\title{
Application of the Pseudo-Transient Technique to a Real-World Unsaturated Flow Groundwater Problem
}

\author{
Fred T. Tracy ${ }^{1}$, Barbara P. Donnell ${ }^{2}$, Stacy E. Howington ${ }^{2}$, \\ and Jeffrey L. Hensley ${ }^{1}$ \\ 1 Information Technology Laboratory, \\ Major Shared Resource Center (MSRC) \\ 2 Coastal and Hydraulics Laboratory, \\ Engineer Research and Development Center (ERDC), \\ Vicksburg, MS, USA 39180
}

\begin{abstract}
Modeling unsaturated flow using numerical techniques such as the finite element method can be especially difficult because of the highly nonlinear nature of the governing equations. This problem is even more challenging when a steady-state solution is needed. This paper describes the implementation of a pseudo-transient technique to drive the solution to steady-state and gives results for a real-world problem. The application discussed in this paper does not converge using a traditional Picard nonlinear iteration type finite element solution. Therefore, an alternate technique needed to be developed and tested.
\end{abstract}

\section{Introduction}

Modeling unsaturated flow using numerical techniques such as the finite element method can be especially difficult because of the highly nonlinear nature of the governing equations. This challenge is even more exacerbated when (1) a steady-state solution is needed, (2) soil properties such as relative hydraulic conductivity go from almost horizontal to almost vertical (see Fig. 1), (3) an influx of water from rainfall occurs at the top of very dry, low hydraulic conductivity unsaturated soil, (4) injection wells are in the unsaturated zone (see IG-1 in Fig. 2), and (5) some pumped wells become totally above the water table or in the unsaturated zone. An early version of the data set for the application discussed in this paper has all of these traits, and the steady-state solution does not converge using a traditional Picard nonlinear iteration finite element solution. The data set needed to be modified to relieve some of the above problems, but a converged solution was needed to know what changes to make. Therefore, an alternate solution was developed and tested. This paper describes the implementation of a modified version of a pseudo-transient technique described in [3] to drive the solution to steady-state and gives computational results for this real-world application. 


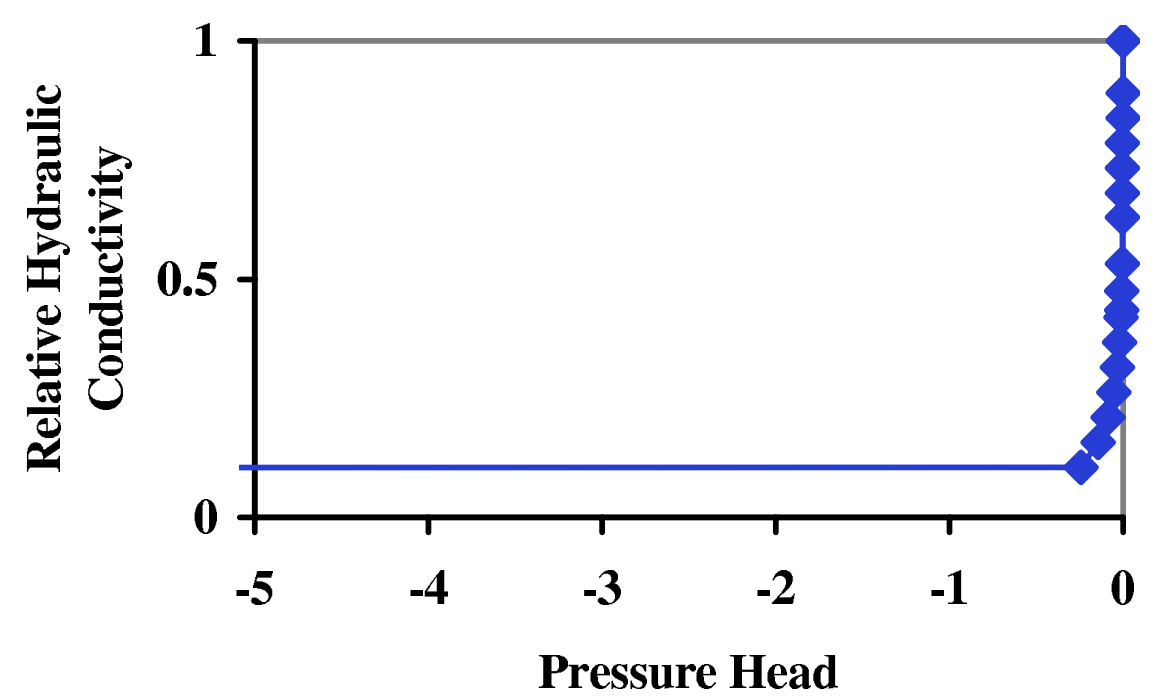

Fig. 1. This figure illustrates the relative hydraulic conductivity versus pressure head curve for soils such as sand where the curve goes from almost horizontal to near vertical rather quickly

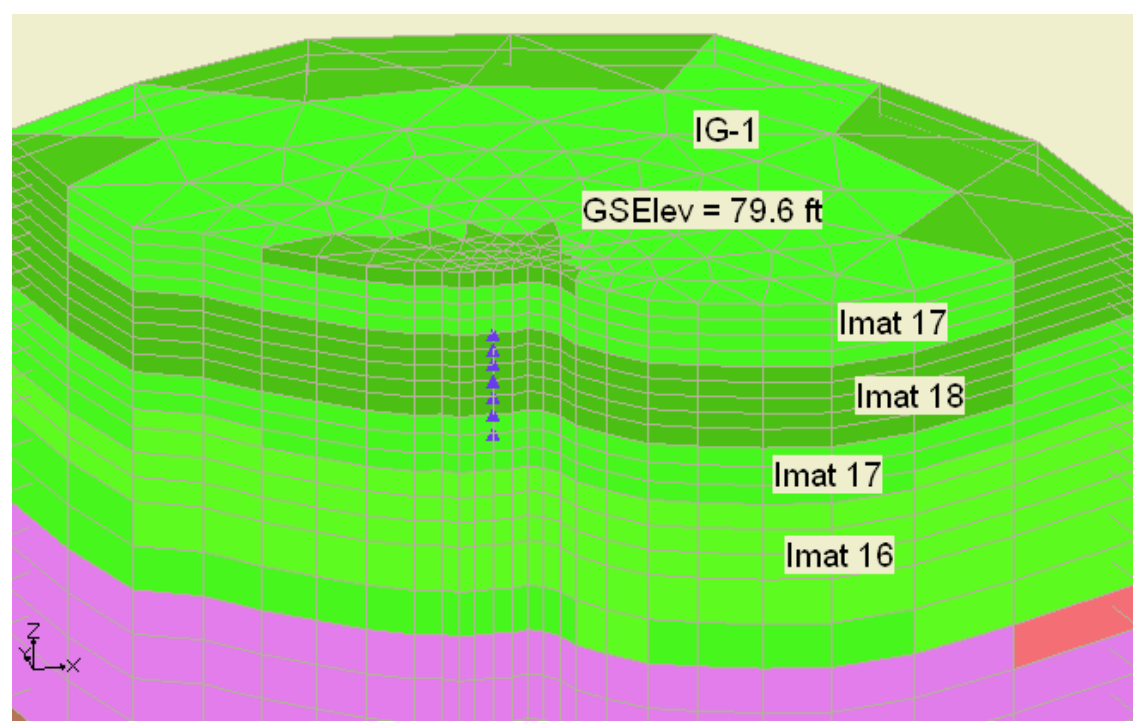

Fig. 2. This figure shows the top twenty layers of the mesh. There are 18 different material types. A large injection well, IG-1, in the unsaturated zone is also shown

The computer model used in this study is the parallel version [7] of FEMWATER [5], which is a groundwater flow and transport code. The finite element mesh is partitioned using METIS [2]. 


\section{Description of the Application}

The problem consists of modeling the remediation of a military site using a pump-and-treat system. The finite element mesh uses 402,628 nodes and 779,328 three-dimensional prism finite elements. There are 18 different soil types, 9 injection wells, 1 chimney drain which also injects water, and 38 extraction wells. Once the model is properly calibrated with observed data, it is then used to predict the efficiency of a proposed pump-and-treat system. Table 1 shows the saturated hydraulic conductivities for the three material types at the top of the ground (16-18 in Fig.2). The incredibly small $k_{z}=0.0001 \mathrm{ft} /$ hour where the ground is very dry and water from precipitation is coming into the system adds significantly to the difficulty of convergence.

Table 1. Saturated hydraulic conductivities (ft/hour)

\begin{tabular}{|c|c|c|c|}
\hline Material Number & $k_{x}$ & $k_{y}$ & $k_{z}$ \\
\hline 16 & 1.042 & 1.042 & 0.008 \\
17 & 0.08 & 0.08 & 0.008 \\
18 & 0.01 & 0.01 & 0.0001 \\
\hline
\end{tabular}

\section{Flow Equations}

Pressure head for steady-state conditions in FEMWATER is modeled by applying conservation of mass to obtain,

$$
\begin{gathered}
\nabla \cdot\left[k_{r}(h) \mathbf{k}_{s} \cdot\left(\nabla h+\frac{\rho}{\rho_{0}} \nabla z\right)\right]+\sum_{m=1}^{N_{s s}} \frac{\rho_{m}^{*}}{\rho_{0}} Q_{m} \delta\left(\mathbf{r}-\mathbf{r}_{m}\right)=0 \\
h=\frac{p}{\rho_{0} g} .
\end{gathered}
$$

where $\delta$ is the Dirac delta function, $g$ is the acceleration due to gravity, $h$ is the pressure head, $k_{r}(h)$ is the highly nonlinear relative hydraulic conductivity, $\mathbf{k}_{s}$ is the saturated hydraulic conductivity tensor, $N_{s s}$ is the number of source/sink nodes for flow, $Q_{m}$ is the quantity of flow at the $m^{\text {th }}$ source/sink node, $p$ is the pressure, $\rho$ is the density with contaminant, $\rho_{0}$ is the density without contaminant, $\rho_{m}^{*}$ is the density of the $m^{\text {th }}$ source/sink fluid, $\mathbf{r}$ is a vector from the origin to an $(x, y, z)$ point in space, and $\mathbf{r}_{m}$ is the location of the $m^{\text {th }}$ source/sink node.

The Galerkin finite element method is then applied to obtain

$$
\mathbf{K}(\mathbf{h}) \mathbf{h}=\mathbf{Q}^{\prime}(\mathbf{h}) .
$$

Here $\mathbf{K}(\mathbf{h})$ is the stiffness matrix, $\mathbf{h}$ is a vector of pressure heads at the finite element nodes, and $\mathbf{Q}^{\prime}(\mathbf{h})$ is a collection of flow type terms for the right-hand side. Eq. 3 is the resulting system of nonlinear equations to be solved. 


\section{Solution of the System of Nonlinear Equations}

A Newton iteration [4] to reduce the nonlinear residual,

$$
\mathbf{F}(\mathbf{h})=\mathbf{Q}^{\prime}(\mathbf{h})-\mathbf{K}(\mathbf{h}) \mathbf{h}
$$

is given by

$$
\begin{gathered}
\mathbf{h}_{n+1}=\mathbf{h}_{n}-\mathbf{F}^{\prime}\left(\mathbf{h}_{n}\right)^{-1} \mathbf{F}\left(\mathbf{h}_{n}\right), \\
\mathbf{F}^{\prime}(\mathbf{h})_{i j}=\frac{\partial(\mathbf{F})_{i}}{\partial(\mathbf{h})_{j}}(\mathbf{h}) .
\end{gathered}
$$

where $\mathbf{F}^{\prime}(\mathbf{h})$ is the Jacobian matrix. FEMWATER uses a Picard iteration, which is equivalent to approximating the Jacobian matrix by $-\mathbf{K}$. This produces

$$
\mathbf{h}_{n+1}=\mathbf{h}_{n}+\mathbf{K}_{n}^{-1}\left(\mathbf{Q}_{n}^{\prime}-\mathbf{K}_{n} \mathbf{h}_{n}\right)=\mathbf{K}_{n}^{-1} \mathbf{Q}_{n}^{\prime} .
$$

The disadvantage of the Picard approximation is the loss of potentially important terms, but the advantage is that the system of simultaneous, linear equations to be solved (Eq. 7) remains symmetric and positive-definite. Thus, a parallel preconditioned conjugate gradient solver works quite well.

\subsection{Convergence of the Steady-State Problem}

Because of factors such as the severe relative hydraulic conductivity curve shown in Fig. 1, the steady-state solution for this data set did not converge. Convergence is helped somewhat by adding a dynamic type relaxation to the Picard iteration as follows:

$$
\begin{gathered}
\mathbf{h}_{n+1}=\left(1-\alpha_{n+1}\right) \mathbf{h}_{n}+\alpha_{n+1} \overline{\mathbf{h}}_{n+1} \quad 0<\alpha_{n+1} \leq 1, \\
\overline{\mathbf{h}}_{n+1}=\mathbf{K}_{n}^{-1} \mathbf{Q}_{n}^{\prime} .
\end{gathered}
$$

where the relaxation factor $\alpha_{n+1}$ is adjusted based on whether the maximum absolute value of the pressure head change $|\triangle h|_{n+1}^{\max }$ between $h_{n}$ and $\bar{h}_{n+1}$ for all the $N$ finite element nodes,

$$
|\triangle h|_{n+1}^{\max }=\max _{i=1}^{N}\left|\left(\overline{\mathbf{h}}_{n+1}\right)_{i}-\left(\mathbf{h}_{n}\right)_{i}\right| .
$$

decreased or increased from $|\triangle h|_{n}^{\max }$. The adjustment used is

$$
\begin{gathered}
\alpha_{n+1}=\min \left(\alpha_{n}+\epsilon_{\alpha}, \alpha_{\max }\right) \quad|\triangle h|_{n+1}^{\max } \leq|\triangle h|_{n}^{\max }, \\
\alpha_{n+1}=\max \left(f_{\alpha} \alpha_{n}, \alpha_{\min }\right) \quad|\triangle h|_{n+1}^{\max }>|\triangle h|_{n}^{\max }, 0<f_{\alpha}<1 .
\end{gathered}
$$

where $\epsilon_{\alpha}, f_{\alpha}, \alpha_{\min }$, and $\alpha_{\max }$ are input variables. Values that worked well for this application are $\epsilon_{\alpha}=0.005, f_{\alpha}=0.667, \alpha_{\min }=0.01$, and $\alpha_{\max }=0.5$. 


\subsection{Pseudo Time-Step Implementation}

For those problems such as the initial data set used in this study where the steady-state Picard iteration would not converge, an additional term is added to Eq. 3 to produce

$$
f^{m+1} \mathbf{M}\left(\mathbf{h}^{m+1}\right)\left(\mathbf{h}^{m+1}-\mathbf{h}^{m}\right)+\mathbf{K}\left(\mathbf{h}^{m+1}\right) \mathbf{h}^{m+1}=\mathbf{Q}^{\prime}\left(\mathbf{h}^{m+1}\right) .
$$

where $f^{m+1}$ is a multiplication factor equivalent to the reciprocal of a time increment for pseudo time-step $m+1, \mathbf{h}^{m}$ is pressure head for pseudo time-step $m$, and after some experimentation, $\mathbf{M}$ was chosen to be the diagonal of $\mathbf{K}$. Adding this term is acceptable because eventually steady-state will be achieved, and thus $\mathbf{h}^{m+1} \approx \mathbf{h}^{m}$, causing the additional term to vanish. The Picard iteration for this implicit Euler approximation now becomes,

$$
\mathbf{h}_{n+1}^{m+1}=\left(f^{m+1} \mathbf{M}_{n}^{m+1}+\mathbf{K}_{n}^{m+1}\right)^{-1}\left(\mathbf{Q}_{n}^{\prime m+1}+f^{m+1} \mathbf{M}_{n}^{m+1} \mathbf{h}^{m}\right) .
$$

To compute $f^{m+1}$, the norm of the residual must first be computed. The residual is computed from

$$
\mathbf{F}_{1}^{m+1}=\mathbf{Q}_{1}^{\prime m+1}+f^{m+1} \mathbf{M}_{1}^{m+1} \mathbf{h}^{m}-\left(f^{m+1} \mathbf{M}_{1}^{m+1}+\mathbf{K}_{1}^{m+1}\right) \mathbf{h}_{1}^{m+1} .
$$

But since the pressure head at time-step $m$ is the same as that of the first nonlinear iteration of time-step $m+1$,

$$
\mathbf{h}_{1}^{m+1}=\mathbf{h}^{m},
$$

Eq. 15 becomes

$$
\mathbf{F}^{m}=\mathbf{Q}^{\prime m}-\mathbf{K}^{m} \mathbf{h}^{m} .
$$

Using the discrete $l^{2}$ norm on $R^{N}$,

$$
\left\|\mathbf{F}^{m}\right\|=\sqrt{\frac{1}{N} \sum_{i=1}^{N}\left(\mathbf{F}^{m}\right)_{i}^{2}},
$$

$f^{m+1}$ is computed by using a version of the switched evolution relaxation (SER) algorithm [6] as follows:

$$
f^{m+1}=f^{m} \frac{\left\|\mathbf{F}^{m}\right\|}{\left\|\mathbf{F}^{m-1}\right\|} .
$$

In most cases, the nonlinear iteration for a pseudo time-step converged in a few Picard iterations. However, occasionally the same instability that caused the need for the pseudo time-step algorithm in the first place generated a lack of convergence after 50 iterations. If that happened, $f^{m+1}$ was modified by

$$
f^{m+1}=f^{m+1}+\frac{1}{2} f^{0} .
$$


every 50 iterations until convergence occurred. $f^{0}$ is computed from the input value of $\triangle t$ by

$$
f^{0}=\frac{1}{\triangle t}
$$

Also,

$$
f^{1}=f^{0}
$$

The advantage of this approach is that each new pseudo time-step brings the solution closer to steady-state, and the added term in Eq. 13 is simpler than the real transient term that could be used. Doing only one nonlinear iteration per pseudo time-step was also tried, but for this application, the instability so dominated that a full convergence of each pseudo time-step seemed best. For this data set, $f^{m+1}$ always became so small that the inherent instability reemerged such that $f^{m+1}$ would become bigger. However, the residual gradually became smaller for most pseudo time-steps. Initially, a number of traditional Picard nonlinear iterations $\left(f^{m+1}=0\right)$ are done, and only if convergence is not achieved the traditional way is the pseudo time-stepping started. This way, only isolated areas in the unsaturated zone need further adjustment.

\section{$5 \quad$ Results}

The application data set was run on the ERDC MSRC SGI 3900 with 32 processors using the material properties given in Table 1 with additional data described in Table 2. Time required to do the computation was 19,593 seconds (5.4425 hour). It was not practical to do a true transient solution with the material properties given in Table 1, as convergence was so difficult, even when increasing the pumping rates and precipitation flux gradually. Thus the material properties were modified as shown in Table 3 to an easier problem. The consequence of changing the material data is that a different problem is solved. It will, however, allow the comparison of errors between a true transient solution and a more difficult problem solved by the pseudo time-stepping technique.

To determine accuracy, six wells in the unsaturated zone are examined for error between the input pumping rates and those produced after the solution process. The true transient solution was run for a simulation time of 288 hours

Table 2. Computational data

\begin{tabular}{|l|c|}
\hline Number of traditional Picard iterations & 1,000 \\
Tolerance for convergence of Picard iterations & 0.0005 \\
Number of pseudo time-steps & 1,000 \\
$\triangle t$ for computing $f^{0}$ & 0.05 \\
\hline
\end{tabular}


Table 3. Modified saturated hydraulic conductivities (ft/hour)

\begin{tabular}{|c|c|c|c|}
\hline Material Number & $k_{x}$ & $k_{y}$ & $k_{z}$ \\
\hline 16 & 1.042 & 1.042 & 0.1042 \\
17 & 0.04 & 0.04 & 0.004 \\
18 & 0.02 & 0.02 & 0.002 \\
\hline
\end{tabular}

Table 4. Errors in well pumping rates

\begin{tabular}{|c|c|c|}
\hline Well Number & Pseudo time-stepping & True transient \\
\hline 1 & $3.12 \mathrm{E}-09$ & $1.52 \mathrm{E}-05$ \\
2 & $-3.22 \mathrm{E}-07$ & $1.20 \mathrm{E}-05$ \\
3 & $-4.67 \mathrm{E}-05$ & $7.39 \mathrm{E}-06$ \\
4 & $4.15 \mathrm{E}-09$ & $4.64 \mathrm{E}-06$ \\
5 & $2.31 \mathrm{E}-09$ & $-1.86 \mathrm{E}-04$ \\
6 & $-6.67 \mathrm{E}-07$ & $2.63 \mathrm{E}-05$ \\
\hline
\end{tabular}

using the relaxed soil properties. Table 4 shows a comparison of the error in the well pumping rates for the two different techniques.

\section{Conclusions}

The pseudo time-stepping algorithm gives an acceptable alternative to achieving a steady-state solution to the highly nonlinear unsaturated flow groundwater problem when convergence is not obtained by traditional methods. Further, automatic determination of the pseudo time-step size is easily found. Results also show that accuracy can be achieved easier than doing a true transient computation. For this application, the pseudo time-stepping algorithm is currently the only way found thus far to achieve a solution because of the complexities of the true transient term which is also highly nonlinear.

\section{Future Work}

As described in [1], alternate approaches will next be investigated. One example is to use Newton iterations with line search after a given number of Picard iterations have been completed. Also, the nonlinear Newton-Krylov solvers described in [8] will be investigated. 


\section{Acknowledgment}

This work was supported in part by a grant of computer time from the Department of Defense High Performance Computing Modernization Program at the ERDC MSRC.

\section{References}

1. Farthing, M.W., Kees, C.E., Coffet, T.S., Kelley, C.T., and Miller, C.T.: Efficient Steady-State Solution Techniques for Variably Saturated Groundwater Flow. Adv. in Water Res., 26(2003), 833-849

2. Karypis, G.: METIS (computer program). http://www.users.cs.umn.edu/ karypis/ metis/, University of Minnesota, Minneapolis, MN (2004)

3. Kawanagh, K.R. and Kelley, C.T.: Pseudo Transient Continuation for Nonsmooth Nonlinear Equations. Draft paper, North Carolina State University, Raleigh, NC (2003)

4. Kelley, C.T.: Solving Nonlinear Equations with Newton's Method, SIAM, Philadelphia (2003), 2

5. Lin, H.J., Richards, D.R., Talbot, C.A., Yeh, G.T., Cheng, J.R., Cheng, H.P., and Jones, N.L.: FEMWATER: A Three-Dimensional Finite Element Computer Model for Simulating Density-Dependent Flow and Transport in Variably Saturated Media. Technical Report CHL-97-12, U.S. Army Engineer Research and Development Center (ERDC), Vicksburg, MS (1997)

6. Mulder, W. and Lebr, B.V.: Experiments with Typical Upwind Methods for the Euler Equations. J. Comp. Phys., 59(1985), 232-246

7. Tracy, F.T., Talbot, C.A., Holland, J.P., Turnbull, S.J., McGehee, T.L., and Donnell, B.P.: The Application of the Parallelized Groundwater Model FEMWATER to a Deep Mine Project and the Remediation of a Large Military Site. DoD HPC Users Group Conference Proceedings, Monterey, CA (1999)

8. Wheeler, M.F., Kle, H., Aksoylu, B., and Eslinger, O.: Nonlinear Krylov Based Solvers. PET Report, University of Texas at Austin, Austin, TX (2005) 